\title{
Determinant of Early Initiation of Breastfeeding in a Tertiary Neonatal Unit
}

\author{
Nepal D', Jeeva SM², Mishra $S^{3}$, Paul VK ${ }^{4}$ \\ ${ }^{1}$ Dr. Deepeshwara Nepal, MD, Senior Medical Officer, Kanti Children's Hospital, Maharajgunj, Kathmandu, Nepal. \\ ${ }^{2}$ Dr. Jeeva Sankar MD, DM Resident in Neonatology. All India Institute of Medical Science, New Delhi, India. \\ ${ }^{3}$ Dr. Satish Misra, MD, DM Resident in Neonatology. All India Institute of Medical Sciences, New Delhi, India. \\ ${ }^{4}$ Dr. Vinod Paul, MD, Ph.D. Professor and Head of Neonatology Unit, All India Institute of Medical Science, New \\ Delhi, India. All affiliated to: WHO Collaborating Centre for Newborn Care and Research, Division of Neonatology, \\ Department of Pediatrics, All India Institute of Medical Sciences, New Delhi, India.
}

Address for Correspondence: Dr. Deepeshwara Nepal, E-mail: dnepal123@hotmail.com

\begin{abstract}
Introduction: Initiation of breastfeeding within one hour of birth is an important determinant of successful breastfeeding. National Family and Health Survey -3(NFHS-3) reported that only $23.4 \%$ of children $<3$ years were breastfed within one hour of birth. Objectives: the purpose of this study is to study the determinant of initiation of breastfeeding within one hour of birth. Setting: Tertiary -level neonatal unit. Material and Methods: All mothers admitted in in the postnatal ward were eligible for inclusion; mothers of sick and /or preterm infants were excluded. Enrolled mothers were interviewed between 24 and 72 hours after delivery. Results: The proportion of mothers who initiated breast feeding within one hour of delivery was 32\%, between 1-6 hrs were $47 \%$ and between 6 to $48 \mathrm{hrs}$ were $21 \%$. Maternal age, education, socioeconomic status, occupation and antenatal or labor room counseling did not influence the initiation of breast feeding within one hour of delivery in univariate analysis. On multivariate analysis, admission in the general ward and delivery by caesarean section were found to be significantly associated with not initiating breastfeeding within one hour (adjusted ORs: 8.79, 2.48 to $31.08, p=0.001$ and $6.79,4.07$ to $22.02 p=0.001$ respectively). Only about $13 \%$ of the infants received prelacteal feeds. Conclusion: Mothers delivering by caesarean section or admitted in the general ward were at high risk of not initiating breastfeeding within one hour. Innovative strategies are required to ensure timely ignition of breastfeeding.
\end{abstract}

Key words: Breastfeeding, Caesarean section, prelacteal feeds, time of initiation.

\section{Introduction}

$\mathrm{B}$ reast feeding is the best way of providing ideal food for the optimal growth \& development of an infant. It also protects babies from common diseases like ARI \&diarrhoeal diseases. It is not only beneficial to the baby but also beneficial to the mother, family, society and a nation as a whole.

Early initiation of breastfeeding has been shown to be associated with a higher prevalence of subsequent breastfeeding ${ }^{1}$. Initiation of breastfeeding within $1^{\text {st }}$ hour of birth is the first and most vital steps towards reducing infant and under five mortality and foremost unacceptably high neonatal mortality rate in developing countries like India and Nepal. According to global data out of all 10.9 million under five deaths 4 million deaths occur Neonatal period. One third of which is due to infection, we can prevent this mortality by one of the cheapest and safest method i.e. by exclusive breastfeeding. According to National family \& health Survey-3(NHFs-3) only $23.4 \%$ neonates were breastfed early in India like wise according to NDHS survey 2006 only $35.4 \%$ of newborn were initiate breastfeeding early in Nepal. 
To enable mothers to establish and sustain exclusive breast feeding for 6 months WHO and UNICEF recommend:

a. Initiation of breast feeding within $1^{\text {st }} \mathrm{hr}$.

b. Exclusive breast feeding.

c. Breast feeding on demand.

d. No use of artificial nipples, pacifier or teats.

While breast feeding is a natural act, it is also a learned behaviour. Extensive research has demonstrated that mothers and other caregivers require active support for establishing and sustaining appropriate breast feeding practices. The decision to breast feeding is influenced by diverse factors including demographic variables, attitude and knowledge, physician endorsement support from the family members ${ }^{2}$ to ensure that expectant mothers make a truly informed decision about infant feeding; prenatal breast feeding education, breast feeding counselling in labour room, postnatal ward and maternity ward should be practised in each step. Extensive researches document the impact of specific health care practices and hospital routines on breast feeding outcomes. Practices that promote successful breast feeding includes early initiation of breast feeding ${ }^{3}$, continuous rooming $\mathrm{in}^{4}$, demand feeding ${ }^{5}$, avoidance of unnecessary formula supplementation ${ }^{6}$, avoidance of artificial nipples ${ }^{7}$, availability of knowledgeable staff $^{8}$, correct breast feeding technique ${ }^{9}$ and appropriate support in the post discharge period.

Despite the documented rational for breast feeding by BFHI, the hospital itself as a barrier for breast feeding remains widespread. Common practices that interfere with successful lactation include delayed initiation of breast feeding, separation of mother and baby, restricted feeding, use of formula feeding, routeings use of pacifiers, insufficient guidance in correct breast feeding techniques and exclusive alliance between hospitals and formula companies. Any maternal or infant factor that limit exclusive breastfeeding should be identified early and solve the problems immediately offer the best chance of successful lactation.

\section{Objective}

To study the determinants of initiation of breastfeeding within one hour of birth.

\section{Material and methods}

This was the Hospital based cross sectional study conducted at Tertiary level neonatal unit of All India Institute of Medical Sciences (AIIMS), New Delhi; in maternity wards including postnatal ward and private wards. A total of 100 mothers who had delivered at AIIMS over a period of two months (May-June 2007) were taken for the study. Informed verbal consent was taken from all mothers. They were interviewed by using standard questionnaire which was pretested. The time of interview ranged from 24-72 hrs of delivery. Data were analysed by using EPI-INFO software. Inclusion criteria: Mothers who gave birth to singleton baby at term gestation. Exclusion criteria: Preterm babies, sick term babies who required NICU admission, severe congenital anomalies and multiple births

\section{Results}

A total of 100 mothers delivering at AIIMS were enrolled in the study. More than half (56\%) of mothers were of 25-29 yrs of age group. Only $2 \%$ of mothers were $>35$ years and none of them were below 20 years. More than one third (34\%) had secondary school education. Almost two thirds (67\%) of mothers were from lower middle class family. Almost half (49\%) of mothers delivered vaginally. Eighty percent of mothers were house wives.

Thirty two percent (32\%) of mothers initiated early breast feeding (within $1^{\text {st }} \mathrm{hr}$. of delivery), $88 \%$ of mothers initiate breast feeding within first $12 \mathrm{hrs} .75 \%$ of mothers were counselled for the early initiation of breast feeding, where as $7 \%$ of mothers never received breast feeding counselling till the time of interview.

Only one third of mothers initiated breast feeding within one hour of birth but more than ninty five percent of mothers start breastfeeding within twenty four hours of delivery.

Almost 30\% had given prelacteal feeding. Among these more than half $(56.6 \%)$ received formula feeding in the form of prelacteal feeding indicating wide use of formula feeding in the hospital. About seventy six (76.6\%) of infants received prelacteal feeding at 1-6 hrs of life, whereas only $16.7 \%$ of neonates received prelacteal feeding within $1^{\text {st }} \mathrm{hr}$ of birth, indicating that there is large scope of avoiding prelacteal feeding by avoidance of giving formula feeding and early initiation of breast feeding by giving early breast feeding counselling and supporting the mother for breast feeding.

Results were analysed and on multivariate analysis: Delivery by caesarean section was found to be significantly associated with not initiating breastfeeding within one hour of birth. (OR 6.79, 4.07 to 22.07 $p=0.001$ ). Like wise on multivariate analysis; admission in general ward was also found to be significantly associated with not initiating breastfeeding within one hour of birth (OR: 8.79, 2.48 to $31.08, p=0.001$ ). All others variables i.e. mother's age, maternal education, maternal occupation and socioeconomic status was insignificantly associated with early initiation of breast feeding. Similarly mothers who underwent caesarean 
section were giving significantly more prelacteal feeding to their babies $(p=0.0001)$. Other factors i.e. maternal age group, maternal education, maternal occupation and socioeconomic condition were not significantly associated for prelacteal feeding. Only two thirds $(66.7 \%)$ of mothers received breastfeeding counselling from doctors during ANC period.

Table1: Showing Demographic Variables of Mothers.

\begin{tabular}{|l|c|}
\hline 1. Maternal Age Group & Percentage \\
\hline $20-24$ & $26(26 \% 0$ \\
\hline $25-29$ years & $56(56 \%)$ \\
\hline $30-34$ years & $16(16 \%)$ \\
\hline$>35$ years & $2(2 \%)$ \\
\hline 2. Maternal Education & Percentage \\
\hline Metrics & $34(34 \%)$ \\
\hline Intermediate & $22(22 \%)$ \\
\hline Graduation & $25(25 \%)$ \\
\hline Postgraduate & $19(19 \%)$ \\
\hline 3.Socioeconomic Status & Percentage \\
\hline Upper-class & $2(2 \%)$ \\
\hline Upper-Middleclass & $4(4 \%)$ \\
\hline Middleclass & $19(19 \%)$ \\
\hline Lower middleclass & $6(6 \%)$ \\
\hline Lower/Poor class & $8(8 \%)$ \\
\hline 4. Mode of Delivery & Percentage \\
\hline SVD & $49(49 \%)$ \\
\hline C-section & $47(47 \%)$ \\
\hline Forceps & $4(4 \%)$ \\
\hline
\end{tabular}

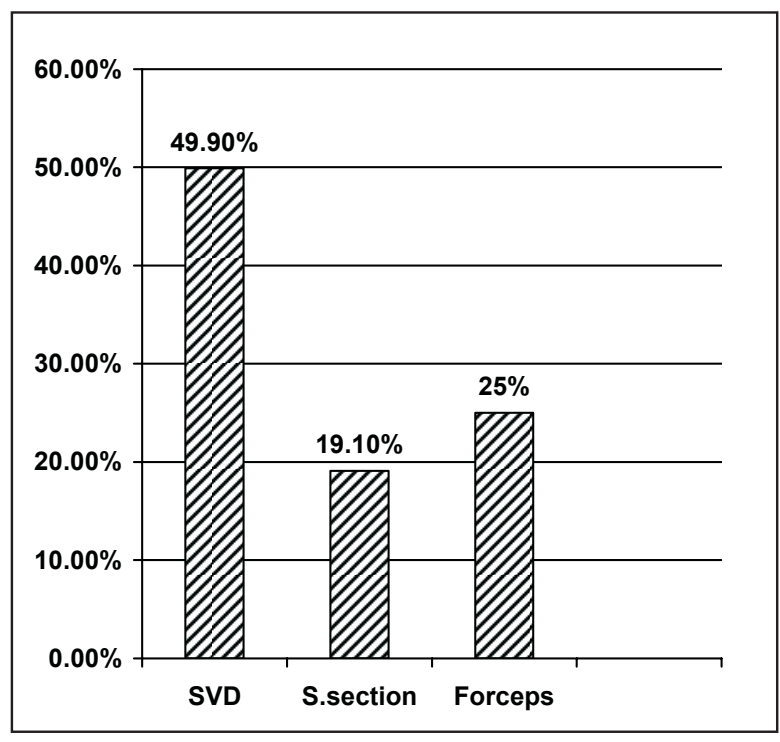

Fig 2: Showing propitiations of Mothers who Initiated Breastfeeding within $1^{\text {st }}$ hour of Delivery According to Mode of Delivery.

\section{Discussion}

In India steps taken for the protection and promotion of the practice of breast feeding have been effective and breast feeding is almost universal. However the message that early initiation of breast feeding, avoidance of prelacteal feeding and exclusive breast feeding up to six months and gradual introduction of semisolid feeds has not been as effectively communicated. Data from literature shows a significant level of exclusive breast feeding only till three months i.e. $55.2 \%$, by six months exclusive breast feeding drop to $19 \%$.goveernment of India is making an extensive effort to promote and support breast feeding. Breast feeding component is incorporated in the national nutrition goals for the $10^{\text {th }} 5$ year plan. The goal for the $10^{\text {th }}$ plan is to:

1. Enhanced early initiation of breast feeding from the current level of $15.8 \%$ (as per NFHS-II) to $50 \%$.

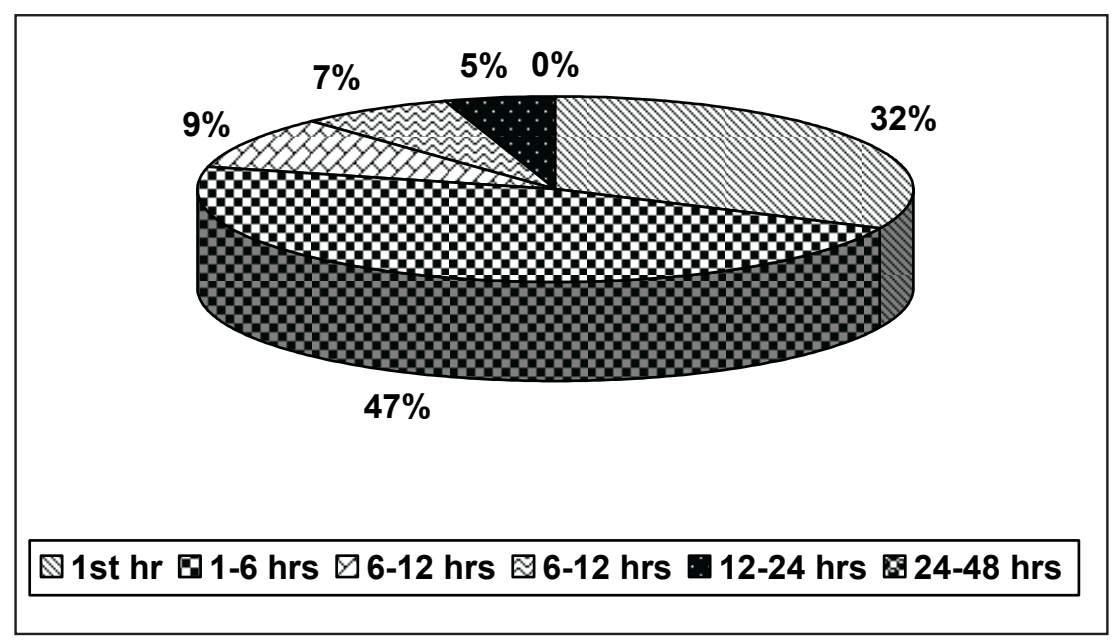

Fig 1: Showing Time of Initiation of Breastfeeding. 
2. Enhanced the exclusive breast feeding rate for children up to the age of 6 months from the current rate of $55 \%$ at 3 months (as per NHFS-II) to 80 $\%$.

In our study $32 \%$ of mothers initiated early breast feeding within $1^{\text {st }} \mathrm{hr}$ of delivery, and $95 \%$ of mothers had initiated breast feeding within $1^{\text {st }} 24 \mathrm{hrs}$ of delivery. Study by Ajay Vatsayan et.al. ${ }^{10}$ in Simla found only $10.3 \%$, but almost similar percentage of mothers (92.21\%) started breast feeding within $1^{\text {st }} 24 \mathrm{hrs}$ of delivery. Kameshwara rao et. al. ${ }^{11}$ in their study which was found to be similar to our study showed $39.5 \%$ of mothers practiced exclusive breast feeding practices. Similar finding have also been observed by Ram et al. ${ }^{12}$ i.e. $85.5 \%$ of infants were put on breast milk within first 24 hours after their birth. Similarly K.S.Negi ${ }^{13}$ observed $80.3 \%$ of the mothers initiated breastfeeding within $7-$ 18 hours after delivery.Taneja ${ }^{14}$ found that exclusive breast feeding was not so common $(26.4 \%)$ as water was started to be given in most infants in the first month itself. So much of a difference in studies regarding early initiation of breast feeding could be due to difference in hospital and community setup.

This study we observed that, caesarean section was found to be a significant cause of delayed initiation of breast feeding $(19.9 \%$ vs. $44.9 \%, p=0.00030)$. A similar type of finding was observed by Vatsayan et.al ${ }^{10}$ also observed similar correlation between caesarean section and a decreased rate of initiation of early breast feeding (only $8 \%$ of mothers undergoing caesarean section initiated breast feeding within $1^{\text {st }} 8 \mathrm{hrs}$ of delivery, $\mathrm{p}=0.000$ ). Similar type of findings was also observed by Pandit N.Yeswant ${ }^{15}$. These findings suggest that more and more support should be given for mothers who underwent caesarean section for early initiation of breast feeding.

No others factors in this study like; maternal age, maternal education, maternal occupation and socioeconomic status were correlated significantly with early initiation of breast feeding. similar finding was observed by Ajaya Vatsayan ${ }^{10}$.The finding that education and breastfeeding counselling of mothers is not conclusively linked to the initiation of breastfeeding may be due to other confounding factors as mother-in -law preferences, delayed rooming in of the child or simply personal attitude despite education.

In our study we found that only $13 \%$ of mothers gave prelacteal feeding. Chhabra et a ${ }^{16}$ also demonstrated from their study that in an urban resettlement colony of Delhi, $76.9 \%$ infants received prelacteal feeding. Devdas et a ${ }^{17}$ also noted $87.9 \%$ nursing mothers gave sugar water, honey mixed with water, milk or jaggary as prelacteal feeding. A study conducted by Anuradha Goyle ${ }^{18}$ also found $96.6 \%$ of mothers used prelacteal feeds. Such differences could be due to difference in study location (This study being hospital based and others being community based) and the presence of more dedicated hospital staff in this hospital.Among $13 \%$ of the prelacteal feeding group, $70 \%$ of mothers gave animal milk, 23\% of mothers gave honey, $7 \%$ of mothers gave ghutti as $1^{\text {st }}$ feeding. Anuradha et.el ${ }^{18}$ found that plain water $(48.3 \%)$ and jaggary with ghee $46.2 \%$ as a form of prelacteal feeding. This shows that there is further scope of increasing early initiation of breastfeeding by further counselling of mothers and their caregivers.

\section{Conclusion}

Despite antenatal and labour room counselling only about one thirds of mothers initiated breastfeeding within $1^{\text {st }}$ hour of delivery. Mothers delivering by caesarean section and admitted in general wards were at high risk of not initiating breastfeeding within the $1^{\text {st }}$ hour. Innovative strategies like provision of breastfeeding counsellors in the hospital setup, constant counselling to mothers and their immediate relatives who take care of baby and mothers; by doctors and nurses are essential for increasing early breastfeeding. As physicians and other medical personnel play an important role in the initiation of early breast feeding and prolongation of exclusive breast, their skill about breast feeding should be upgraded by giving periodic trainings and conducting workshops. Cent percent of pregnant ladies should get antenatal breast feeding counselling in the ANC period itself, similarly they should be counselled and supported in each and every step regarding breast feeding. As most of the mothers are giving formula feeding as a prelacteal feedings, it should be discouraged.

\section{Acknowledgement}

I wish to thank Dr.Pradeep Sharma, Miss Jyoti Singh, Dr. Ashok Deorari and Dr.Ramesh Aggarwal for their constructive criticism and cooperation throughout the process of my research activity. At last but not the least I would like to thank those newborn and their mothers for cooperating with us and giving me this opportunity.

\section{Funding: None}

\section{Conflict of Interest: None}

\section{References}

1. Taylor PM, Maloni JA, Brown DR. Early Suckling and Prolonged Breastfeeding. Am J Dis Child 1986; 140:151-154.

2. Jacobson SW, Jacobson JL, Frye KF. Incidence and Correlate of Breastfeeding in Socioecnomically Disadvantaged Women. Pediatrics 1991; 88:728732 . 
3. Kurinij N, Shiono PH: Early Formula Supplementation of Breastfeeding. Pediatrics 1991; 88:745-748.

4. Yamauchi Y, Yamanuachi I: The Relationship between Rooming-in/not Rooming in and Breastfeeding Variables.Acta pediatr scand 1990; 79: 1917-1922.

5. Yamauchi Y, Yamanuachi I: Breastfeeding Frequency during the first 24 hours after Birth in Full-Term Neonates. Pediatrics 1990; 86:7377.41 .

6. Perez-Escamilla R, Segura-Mills, Canahauti J, et al: Prelacteal Feeds are Negatively Associated with Breastfeeding outcomes in Hondurus: J Nutri 1996;126:2765-2769

7. Barros FC, Victora CTG, Sewer TC et al. Use of Pacifier is Associated with Decreased Breastfeeding Duration. Pediatrics. 1995; 95:497-481.

8. Powers N, Naylor A, Wester R. Hospital Policies: Crucial to Breastfeeding Success. Seminar Perinatol 1994; 18:517-521.

9. Righard L, Alade MO: Sucking Technique and its Effect on Success of Breastfeeding at Birth. Pediatrics 1992; 19:185187.

10. Vatsayan A, Gupta AK, Dhadwal D,Aluwalia SK, Sharma R, Sood RK. Age during Breastfeeding and timely Suckling. Indian J pediatr 1996;63:791794
11. Rao K, Swaminathan MC, Swarup S, Patwardhan VN. Protein Malnutrition in South India. Bulletin WHO 1989;20:603-609

12. Ram R, Ghosh MN, Saha JB, Bhattacharya SK, Biswas AH, Chattrjee C. Breastfeeding Practices in the Rural Community of District Darjelling,West Bangal. Ind Jour community Med.2000; 25(2):935.

13. Negi K.S., Kandpal.Breastfeeding Practices in a Rural area of District Deheradun, Uttaranchal. Indian J.Prev.soc.Med.2004; 35:184-8.

14. Taneja DK, Mishra A.Mathur NB. Infant Feeding: An Evaluation of Text and Taught. Indian J Pediatr 2005 feb,72(2):127-9

15. Pandit N, Yaswantah M, Albureque Ida. Factors Influencing Initiation of Breastfeeding in an Urban Setup. Indian Pediatr 1994;31(12)1558-1562

16. Chhabra P, Grover VL, Aggrawal OP, Dubey KK. Breastfeeding Pattern in an Urban Resettlement Colony of Delhi. Indian J Pediatr 1998; 65:867-2.

17. Devdas RP, Purushothaman V, Paul M. Trends in Breastfeeding Practices. Ind J Nutr Dietet 1999; 36:111-4.

18. Goyle A, Jain P, Vyas S, Saraf H, Sekhawat N. Colostrum and Prelacteal Feeding Practices followed by Families of Pavement and Roadside Squatter Settlement. Indian J. prev soc med 2004; 35 (1\&2):58-2. 